\title{
Nouvelles perspectives en chimiothérapie anti-rétrovirale
}

L'élaboration de stratégies thérapeutiques contre les virus HIV exige une bonne connaissance de leur cycle infectieux, de leur pathogénicité et du contrôle de l'expression de leur génome. En théorie, les cibles des chimiothérapies antivirales sont tous les systèmes indispensables au cycle viral ou à l'expression du pouvoir pathogène. Outre les analogues des nucléotides utilisés pour inhiber la transcriptase inverse (dont le type est l'azidothymidine), des inhibiteurs de peptides codés par le génome viral et intervenant dans la maturation et l'activation des particules virales constituent des pistes intéressantes.

\section{Jean-Luc Darlix}

\section{ADRESSE}

J.-L. Darlix : professeur de biochimie à l'université Paul-Sabatier. Labo Rétro, centre de recherche de biochimie et de génétique cellulaires, CNRS, 118, route de Narbonne, 31062 Toulouse Cedex, France.

$\mathrm{m} / \mathrm{s} n^{\circ} 4$ vol. 5 , auril 89

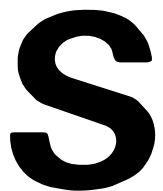

IDA, voilà un mot nouveau de lâché, un mot qui fait fureur, un mot médiatique, mais un mot qui fait peur. SIDA, et voilà ce diable de sigle qui révèle un terme vieux et confidentiel, celui de rétrovirus, au public. Il y a dix ans, peu de biologistes connaissaient cette catégorie de virus appelée rétroviridae, malgré la découverte de l'activité transcriptase inverse en 1970 [1, 2]. Mais aujourd'hui, la biotechnologie, avec ses approches de biologie moléculaire des organismes eucaryotes, serait très inconfortable sans cette ADN polymérase virale. Les premiers rétrovirus furent découverts au début du $\mathrm{xx}^{\mathrm{e}}$ siècle et parmi eux on peut citer le virus du sarcome de Rous (RSV)[3]. Ce virus RSV infecte les oiseaux et peut provoquer certaines formes de cancer chez les animaux infectés.

Un autre virus très connu des rétrovirologistes est le virus de la leucémie murine (MuLV), qui peut induire différentes formes de leucémies chez l'animal. Avant la découverte du virus HIV (human immunodeficiency virus) du SIDA [4] les rétrovirus des types RSV et MuLV ont fait l'objet d'une somme impression- nante de travaux, et n'oublions pas qu'ils furent à l'origine de la découverte de gènes impliqués dans la régulation du cycle cellulaire, les oncogènes [5]. Dans ces conditions, les études sur le virus HIV ont bénéficié, de façon très fructueuse, des connaissances de base déjà acquises en rétrovirologie [6]. Par un juste retour des choses, c'est aujourd'hui, grâce au virus HIV, qu'un grand élan est donné à l'étude des rétrovirus.

Le premier but de cet effort est de mieux connaître le virus HIV et les rétrovirus en général afin de trouver une parade aux conséquences, dramatiques pour le moment, d'une infection par un virus de type HIV. Malheureusement, rares ont été les essais de vaccination contre des rétrovirus, et la vaccination des chats contre le virus de la leucémie féline (FeLV) est le seul exemple de protection vaccinale, bien que partielle, contre un rétrovirus. La chimiothérapie anti-rétrovirale est, hélas, à un stade encore moins avancé. En réalité, ses premiers pas datent de plus de dix ans avec la découverte de drogues qui peuvent blaquer certaines étapes précoces de l'infection par un rétrovirus [7] ou la formation 
de virus infectieux [8]. Bien que ces drogues s'avèrent efficaces in vitro ou ex vivo, elles sont soit cytotoxiques, soit faiblement efficaces in vivo, et le SIDA [7] associé à l'infection par le rétrovirus HIV demeure incurable [9].

Même si ce constat semble sombre, il ne faut pas oublier que la chimiothérapie anti-rétrovirale en est à ses débuts. Naïvement, nous voudrions tous disposer d'un agent qui bloque in vivo la réplication de HIV et soit sans conséquences graves pour la personne traitée. Ce serait l'équivalent d'un antibiotique vis-à-vis d'une infection bactérienne. De plus cet agent devrait être commode et d'un emploi facile.

Alors, face à ces nécessités et grâce à nos connaissances actuelles de la réplication des rétrovirus, et en particulier du virus HIV, comment peut-on définir les objectifs réalistes d'une chimiothérapie anti-rétrovirale? A côté des approches existantes, existe-t-il de nouvelles approches anti-rétrovirales?
Le cycle réplicatif du virus HIV et quelques-uns de ses contrôles

Afin d'essayer de répondre aux questions posées, il est nécessaire de rappeler sommairement la structure génétique de HIV et son cycle réplicatif (figures 1 et 2)[10-12]. N'oublions pas aussi que les traits majeurs de ce cycle sont communs à tous les rétrovirus, et qu'également pas plus de $0,1 \%$ des particules HIV sont infectieuses. Le provirus HIV a la structure rétrovirale type, c'est-àdire deux longues répétitions terminales (LTR, long terminal repeat) encadrent un total actuel de neuf gènes. Les gènes gag, pol et env codent pour les protéines du virion, deux gènes en morceaux, tat et rev, et deux autres, vif et nef, commandent des protéines de régulation, et enfin les deux derniers, $v p u$ et $v p r$, ont une fonction encore inconnue (figure 1).

L'interaction virus-cellule. Les glycoprotéines de surface SU du virion, SU gp 120, reconnaissent les récep- teurs CD4 à la surface de la cellule cible, le lymphocyte T4[11]. La fusion de l'enveloppe virale et de la membrane cellulaire intervient sans doute grâce aux molécules de glycoprotéine TM-gp4l ( $\mathrm{NH}_{2}$ hydrophobe) et à celles de la protéine myristilée MA-pl7, ce qui permet à la capside virale d'entrer dans le cytoplasme (figure 2). La capside ou noyau viral a une structure en obus (environ $600 \AA$ de long sur $300 \AA$ de large) et est formée de protéines abondantes, CA p24 et NC pl5 du gène gag, de l'ARN génomique dimérique (deux copies identiques et liées), et des enzymes transcriptase inverse (RT) p5l-p66 et intégrase (IN) p32 du gène pol.

La synthèse du provirus HIV se fait probablement, et par analogie avec le rétrovirus murin MuLV, dans la capside [13]. La biosynthèse de l'ADN proviral double brin résulte de la copie de l'ARN génomique simple brin en ADN complémentaire par la RT p5l-p66, puis de ce dernier par la même enzyme (figure 2). C'est, sans

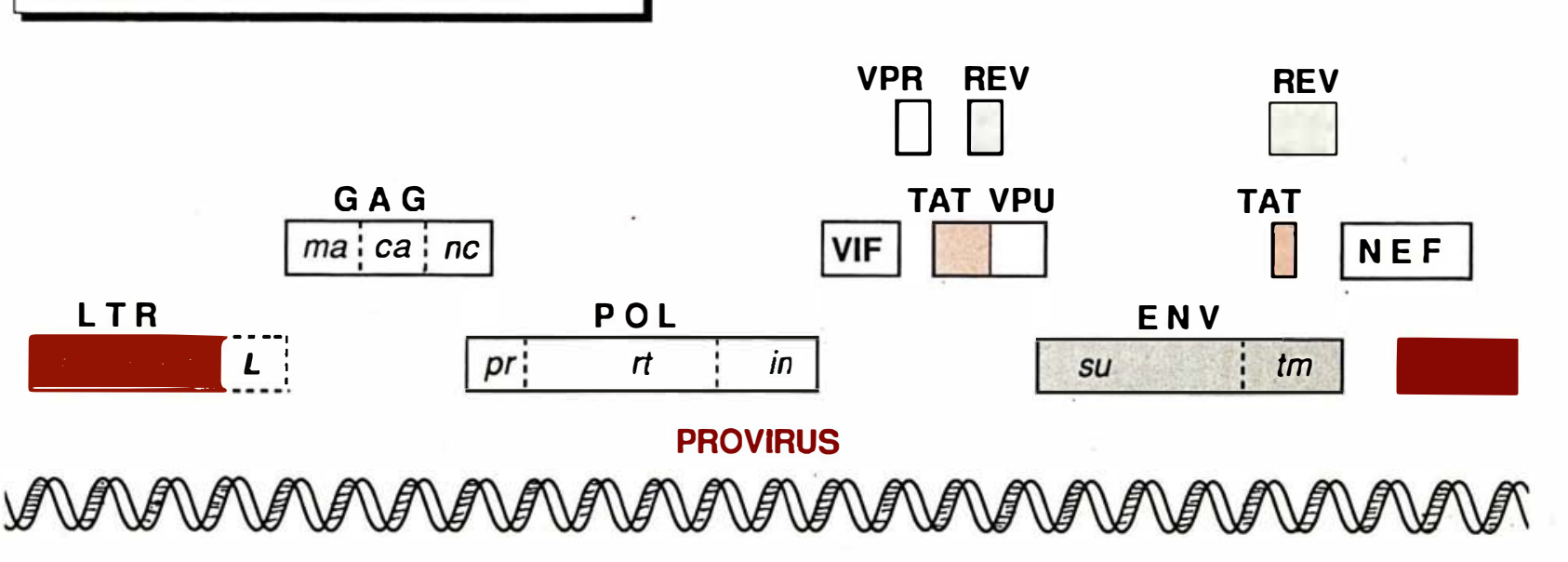

Figure 1. Structure génétique du provirus HIV. Les séquences de contrôle de l'expression, appelées « longues répétitions terminales " (LTR), encadrent neuf gènes dont huit sont identifiés à ce jour. La séquence " $L$ " est unique et est nécessaire à la synthèse du provirus comme à celle des ARN messagers sous-génomiques. " $L$ " contient aussi des séquences requises pour la production des polyprotéines Prgag et Prgag-pol. Le gène gag code pour les protéines de structure du virus HIV (les lettres grasses indiquent le sigle): matrice p17, capside p24 et nucléocapside p15; le gène pol code pour les enzymes qui interviennent au cours du cycle réplicatif: protéase p12, transcriptase inverse p51-p66 (rt : reverse transcriptase) et intégrase p32 (figure 2); le gène env pour les glycoprotéines de surface gp120, qui permet l'attachement du virus au récepteur cellulaire CD4, et transmembranaire gp41 (figure 2). Les gènes tat, rev et vif interviennent sans doute dans le réveil et la dissémination du virus HIV, alors que nef le maintiendrait en latence. La fonction de vpu et vpr n'est pas encore connue. 
doute, sous cet état de complexe nucléoprotéique que l'ADN proviral HIV migre jusqu'au noyau de la cellule pour s'intégrer au génome de l'hôte [3, 6].

L'intégration du provirus HIV nécessiterait l'état nucléoprotéique de l'ADN proviral, l'enzyme IN p32 et une division cellulaire. Dès que le provirus HIV s'est intégré au génome de la cellule (figure 2), celle-ci est définitivement infectée et le provirus HIV se reproduit en même temps que les gènes de l'hôte.

L'expression du provirus HIV dépend du type, et de l'état de la cellule infectée. Ainsi l'expression de HIV dans les lymphocytes T4, et donc la production de nouvelles particules virales, n'est maximale que si les cellules se divisent. La première étape de l'expression est la synthèse de l'ARN génomique $35 \mathrm{~S}$ par transcription du provirus HIV (figure 2). Le taux de synthèse de l'ARN $35 \mathrm{~S}$ est contrôlé par des protéines codées par HIV : la Nef p27 inhibe la transcription du provirus, alors que la Tat pl4 stimule fortement l'expression de HIV (figure 2). Comme chez tous
Figure 2. Schéma du cycle réplicatif de HIV. Les étapes sont numérotées de 1 à 9. (1) Le virus HIV se fixe à la cellule à la suite de la reconnaissance des SU gp 120 et des CD4. (2) Le virus est internalisé par fusion de l'enveloppe virale et de la membrane cellulaire (TMgp41), et la capside entre dans le cytoplasme. (3) La synthèse du provirus par transcription inverse de l'ARN génomique intervient dans la capside. (4) L'ADN proviral sous forme de complexe nucléoprotéique pénètre dans le noyau de la cellule et s'intègre au chromosome de l'hôte (cette intégration nécessite une division cellulaire). (5) Le provirus est transcrit par l'ARN polymérase $B$ de l'hôte, ce qui engendre I'ARN 35S ; la protéine Tat stimule cette expression alors que Nef I'inhibe (signes + et -). (6) L'ARN $35 S$ migre du noyau au cytoplasme et l'équilibre entre les différents ARN viraux est assuré par la protéine Rev d'une part, et des domaines génétiques cis de I'ARN $35 S$ (CAR et CRS); (6a) LARN $35 S$ non épissé est le messager codant pour les polyprotéines Prgag et Prgag-pol. L'ARN 35S est épissé, ce qui engendre toute une série d'ARN messagers qui codent pour la polyprotéine Env (6b), et les protéines Rev (6c). Tat (6d) et Nef (6e). (7). La polyprotéine Env passe par le réticulum endoplasmique, où elle est glycosylée et clivée en SU gp120 et TM gp41. (8). Les polyprotéines Gag et Gag-Pol s'agglomèrent à la face interne de la membrane (8a), s'associent aux glycoprotéines (8b), puis I'ARN 35 S est encapsidé probablement par la NC p15. (9). Les virus immatures quittent la cellule infectée par bourgeonnement, et deviennent matures sous l'effet de la protéase. En même temps, I'ARNt Lys, initiateur de la transcription inverse, est sans doute hybridé à I'ARN $35 S$ (séquence * $L *$ ) par la NC p15.

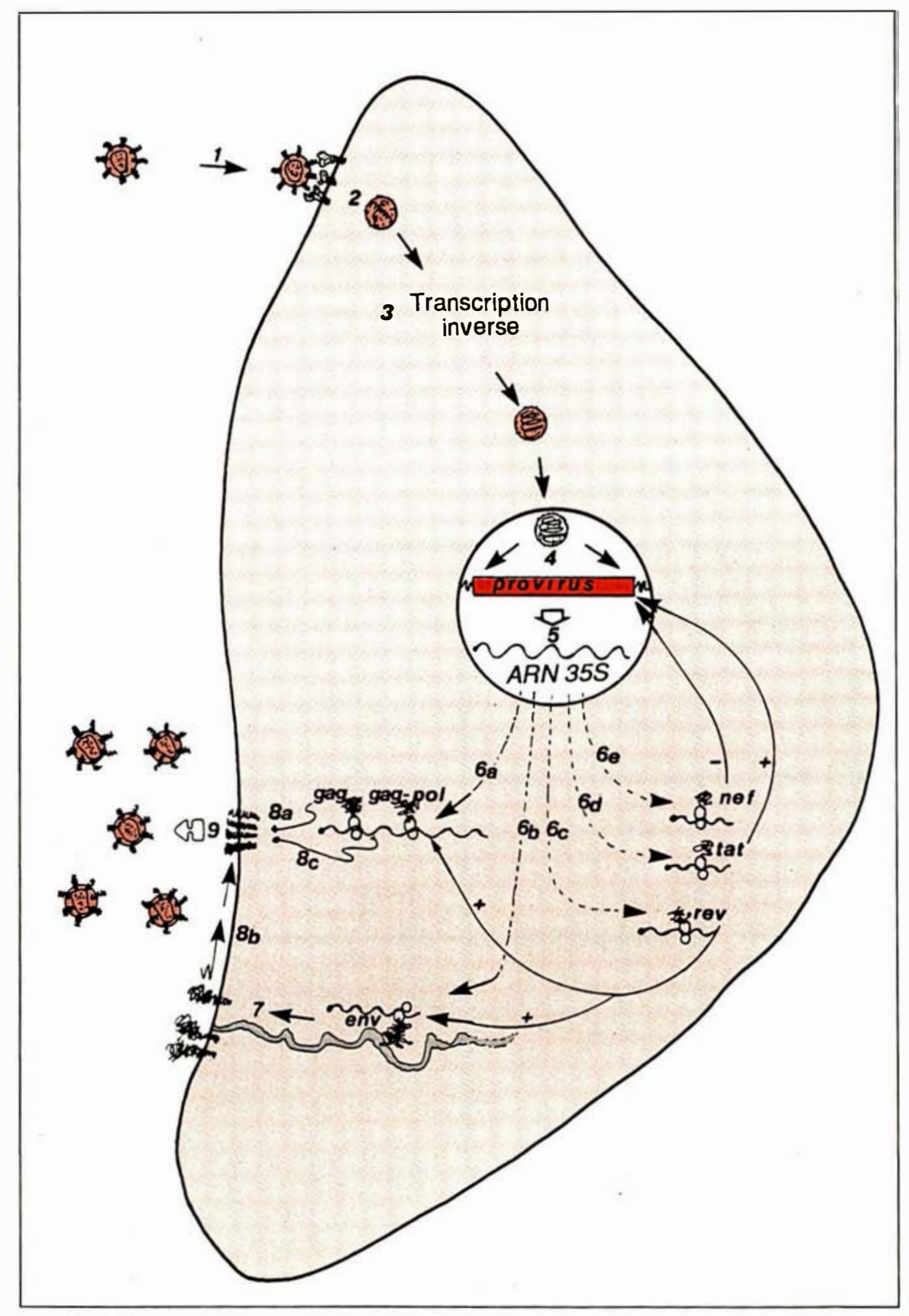




\section{RÉFÉRENCES}

1. Baltimore D. RNA-dependent DNA polymerase in virions of RNA tumor viruses. Nature 1970 ; 226 : 1209-11.

2. Temin HM, Mizutani S. RNA-directed DNA polymerase in virions of Rous sarcoma virus. Nature $1970 ; 226$ : 1211-3.

Le lecteur pourra se reporter aux deux volumes consacrés aux rétrovirus : $i c i, i l$ s'agit de : Taxonomy of Retroviruses p. 25-207.

3. Weiss $\mathrm{R}$, Teich $\mathrm{N}$, Varmus $\mathrm{H}$, Coffin J RNA tumor viruses. Cold Spring Harbor, New York (USA) : Cold Spring Harbor laboratory, 1984.

4. Barré-Sinoussi F, Cherman JC, Rey F, et al. Isolation of $\mathrm{T}$-lymphotropic retrovirus from a patient at risk for acquired immunodeficiency syndrome (AIDS). Science 1983; 220 : 868-70.

5. Bishop JM, Varmus $H$. Functions and origins of retroviral transforming genes. In Weiss R, Teich N, Varmus H, Coffin J, eds. RNA tumor viruses. Cold Spring Harbor New York (USA) : Cold Spring Harbor laboratory, $1984:$ :999-1 100.

6. Varmus H, Swanström R. Replication of retroviruses. In : Weiss $\mathrm{R}$, Teich $\mathrm{N}$, Varmus $\mathrm{H}$, Coffin J, eds RNA tumor viruses. Cold Spring Harbor, New York (USA): Cold Spring Harbor laboratory, 1984 : 369-500.

7. Mitsuya H, Broder S. Strategies for antiviral therapy in AIDS. Nature $1987 ; 325: 773$. 8

8. Levin JG, Rosenak MJ. Synthesis of MuLV proteins associated with virions assembled in actinomycin-D treated cells evidence for persistence of viral messenger RNA. Proc Natl Acad Sci USA 1976; 73 : 1154-8.

9. Dournon E, Rozenbaum W, Michon C, et al. Effects of zidovudine in 365 consecutive patients with AIDS or AIDS related complex. Lancet 1988 ; 1297-302.

10. Sur le sujet du virus HIV et du SIDA, le lecteur peu informé pourra se reporter avec profit au numéro spécial « SIDA » de la revue Pour la Science, décembre 1988.

11. Fauci AS. The human immunodeficiency virus: infectivity and mechanisms of pathogenesis. Science 1988 ; 239 : 617-22.

12. Varmus $H$. Regulation of HIV and HTLV gene expression. Genes Dev 1988; 2 1055-62.

13. Brown PO, Bowerman B, Varmus $\mathrm{H}$, Bishop JM. Correct integration of retroviral DNA in vitro. Cell $1987: 49: 347-56$.

14. Wilson W, Braddock M, Adams SE, et al. (1988). HIV expression strategies : Ribosomal frameshifting is directed by a short sequence in both mammalian and yeast systems. Cell $1988: 55: 1159-69$ les rétrovirus, l'ARN primaire $35 \mathrm{~S}$ est épissé au cours du passage noyaucytoplasme, afin d'engendrer toute une catégorie d'ARN messagers viraux dits «sous-génomiques». Pour que de nouvelles particules virales HIV soient produites, il faut maintenir un équilibre subtil entre l'ARN 35S et les ARN sous-génomiques. En effet l'ARN 35S code pour les protéines de structure et les enzymes du virus HIV, alors que les ARN sous-génomiques codent pour les glycoprotéines de l'enveloppe du virus et les protéines de régulation de l'expression de HIV. Cet épissage partiel et multiple de l'ARN 35S semble être contrôlé par des séquences de l'ARN 35S, appelées CRS et CAR, et par une protéine virale, la Rev pl2.

La traduction des ARN viraux par les ribosomes est très active et plus de $1 \%$ des protéines de la cellule infectée sont virales. L'ARN $35 \mathrm{~S}$ code pour les précurseurs polyprotéiques appelés $\operatorname{Pr} 55^{\text {gag }}$ et $\operatorname{Pr} 180^{\text {gag.pol }}$ qui engendreront les protéines MA pl7, CA p24 et NC pl5 d'une part, et les protéines PR pl2, RT p5l-p66 et IN p32 d'autre part. Les trois premières sont des protéines de structure du virion, et donc abondantes, alors que les trois dernières ont des activités enzymatiques et sont rares dans les virions HIV. Ce déséquilibre dans l'expression des gènes gag et pol est sous un contrôle traductionnel par un mécanisme dit de «glissement ribosomique» au niveau d'une courte séquence de résidus uridine [14]. Les ARN HIV sous-géno- miques codent pour les protéines de régulation, Tat pl4, Rev pl2, Nef $\mathrm{p} 27$, et pour les protéines Vif et Vpu, dont on connaît mal la fonction, et enfin pour le précurseur polyprotéique Prenv. Ce dernier subit une maturation en deux glycoprotéines qui forment l'enveloppe du virus HIV, la SU gpl20 et la TM gp4l (figure 2).

L'assemblage des protéines virales et la formation de virus HIV sont des événements complexes qui interviennent sans doute sous la membrane de la cellule infectée. En effet on peut supposer que les protéines de la capside s'assemblent, puis s'associent aux protéines de l'enveloppe, et qu'enfin le génome ARN est encapsidé. La dynamique pourrait être la suivante:

(a) grâce à des propriétés d'auto-affinité et à la présence d'un acide gras en $\mathrm{NH}_{2}$ terminal, les polyprotéines Pr gag et Prgag-pol s'assemblent sous la membrane; (b) la présence de l'acide gras favoriserait les interactions du Prgag avec les glycoprotéines SU gpl20 et TM gp4l; (c) pour former un virion complet, il y manque le matériel génétique, l'ARN génomique HIV. Le processus d'encapsidation doit choisir l'ARN génomique au milieu des ARN cellulaires et des ARN viraux sous-génomiques. Comment? Pour d'autres rétrovirus, une séquence d'encapsidation appelée «Psi » ou « $\mathrm{E}$ » est connue et détermine l'encapsidation préférentielle de l'ARN génomique viral au détriment de tout autre ARN $[15,16]$. Cette

Tableau I

FONCTIONS DES PROTÉINES CODÉES PAR LES GĖNES DU VIRUS HIV

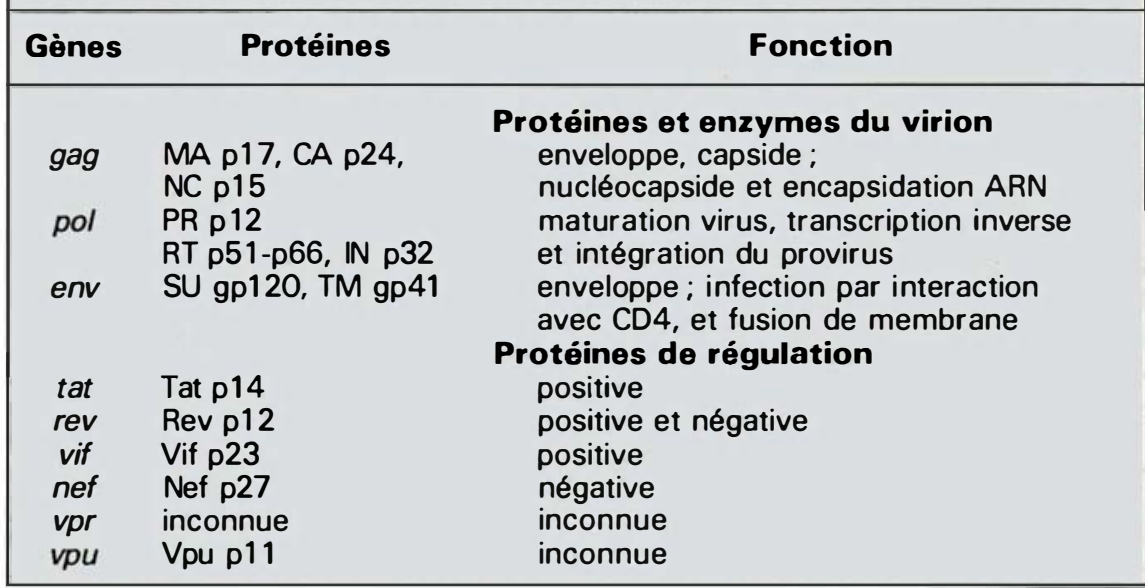




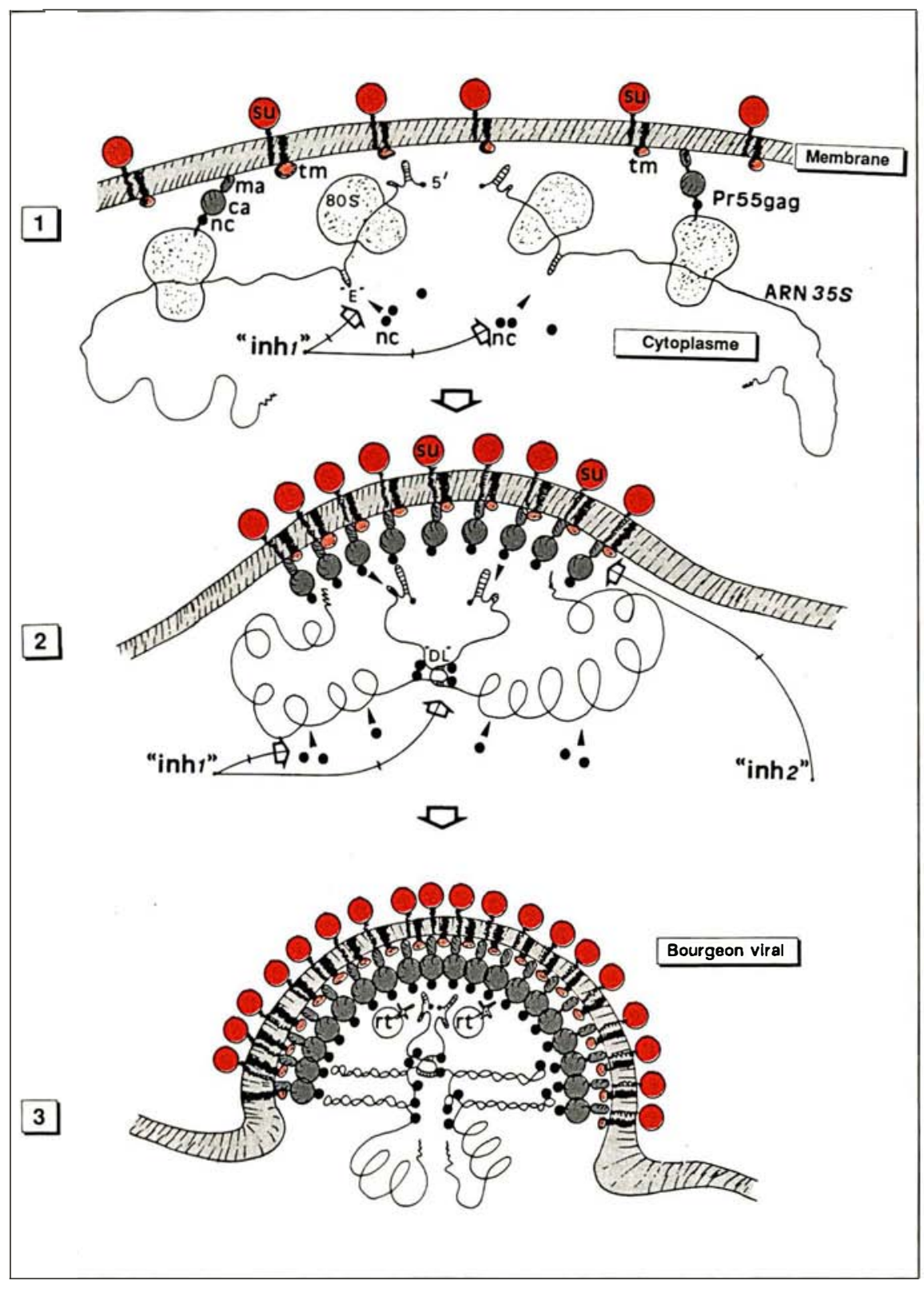

séquence dirigerait l'encapsidation en conduisant l'auto-association de deux molécules d'ARN génomique pour former un $A R N$ génomique dimérique (la figure 3 présente un schéma de l'encapsidation de l'ARN génomique). Une protéine virale semble nécessaire pour induire ce changement conformationnel sans lequel l'ARN viral ne peut pas être encapsidé, c'est la protéine NC pl5[17, 18]. Selon nos plus récentes études, la NC pl 5 conduirait aussi l'encapsidation d'un autre ARN sans lequel le virus HIV n'est pas infectieux, il s'agit de l'ARNt ${ }^{L . y s}$ $\mathrm{m} / \mathrm{s} n^{\circ} 4$ vol. 5 , avril 89 amorce de la réplication de HIV par la transcriptase inverse; (d) les particules virales sortent de la cellule en bourgeonnant et à chaque instant environ $1 \%$ de la surface cellulaire est occupé par des bourgeons en formation. Les virus partent alors avec un fragment de membrane cellulaire, mais ne sont que peu ou pas infectieux, car seule une faible fraction des Prgag et Prgag-pol a subi la maturation complète ; (e) la maturation des précurseurs polyprotéiques par la protéase PR pl2 [19] active les virions, ce qui engendre des virus HIV infectieux (cette maturation est

Figure 3. Schéma de la formation du bourgeon viral et de l'encapsidation de I'ARN génomique HIV par la protéine NC p15. (1) LARN $35 S$ HIV (seul ARN représenté) est traduit par les ribosomes $80 S$ en Pr55gag et Pr180gag-pol (ce dernier n'est pas représenté). Une partie des polyprotéines subit une maturation et c'est probablement ainsi que des molécules de NC p15 apparaissent (ronds noirs). Le Prenv est ancré dans la membrane cytoplasmique (SU et TM). Les NC p15 vont interagir avec I'ARN $35 S$ HIV et entre elles (petites flèches noires). Un inhibiteur de la NC p15 / inh 1 \%) empêche ces interactions. Les sigles définissant les protéines virales sont ceux de la figure 1. (2) Les polyprotéines Prenv, Prgag et Prgag-pol s'agglomèrent sous et dans la membrane cytoplasmique. Les interactions NC p15-ARN $35 S$ conduisent à la dimérisation de I'ARN génomique HIV ( $\propto D L \cdots)$, puis au départ des ribosomes $80 S$ de I'ARN 35S. C'est sans doute grâce à cette modification conformationnelle que I'ARN HIV est reconnu parmi la foule des ARN cellulaires et des quelques ARN viraux, puis empaqueté dans le virion en formation. L'inhibiteur "inh1" empêche cette encapsidation en bloquant la NC p15. L'inhibiteur "inh2» bloque la PR p12 et donc la maturation ultérieure des virions (grosses flèches). (3) Le bourgeon viral apparaît à la surface de la cellule, et l'encapsidation et la condensation de I'ARN HIV dimérique se poursuivent sous l'action de la NC p15. La transcriptase inverse (RT) est déjà présente dans le bourgeon et interagit avec l'ARNtLys, initiateur de la transcription inverse. Plusieurs processus biochimiques vont activer le virion pour donner un virus HIV infectieux: I'hybridation de I'ARNtLys à l'ARN HIV dimére est contrôlée par la NC p15 et peut être inhibée par «inh1 „; la maturation des Prgag et Prgag-pol est assurée par la protéase PR p12 et peut être inhibée par « inh2 $ఎ$; la maturation du Prenv en SU gp120 et TM gp41 est sans doute assurée par des protéases cellulaires.

lente chez HIV). Pendant ce temps, l'ARN L Lys amorce de la réplication serait hybridé au site d'initiation de la réplication de HIV par la protéine NC pl5 [17, 18]. Sans cette dernière étape, l'ARN génomique viral ne peut pas être reproduit en provirus. Cette dynamique de la formation des particules HIV montre qu'au moins deux protéines virales jouent un rôle clé dans l'activation des virions: d'une part, la NC pl5 et son activité hybridase et, d'autre part, la protéase PR p12. Elles semblent donc être des cibles de choix pour une nouvelle chimiothérapie anti-rétrovirale. 


\section{RÉFÉRENCES}

15. Mann R, Baltimore D. Varying the position of a retrovirus packaging sequence results in the encapsidation of both unspliced and spliced R NAs. J Virol 1985 ; 54 : 401-7.

16. Prats AC. Thèse de virologie: Étude de l'expression et de la constitution des particules virales infectieuses chez le rétrovirus murin MuLV. 1988.

17. Prats AC, Sarih L, Gabus C. Litvak S Keith G, Darlix JL. Small finger protein of avian and murine retroviruses has nucleic acid annealing activity and positions the replication primer $\mathbf{T N A}$ onto genomic RNA. EMBO J, $1988 ; 7: 1777-83$.

18. Lullien V, Darlix JL (résultats non publiés).

19. Seelmeier S, Schmidı H, Turk V, von der Helm K. HIV has an aspartic protease that can be inhibited by pepstatin A. Proc Natl Acad Sci (USA) 1988; $85: 6612-6$.

20. Traunecker A, Lüke W, Karjalainen $K$. Soluble CD4 molecules neutralize HIV type 1. Nature $1988 ; 331: 84-6$.

21. Gartner S, Markovits P, Markovits DM, et al. The role of the mononuclear phagocytes in HTLV III/LAV infection. Science 1986 ; $233: 215-9$.

22. Till MA, Ghetie V, Gregory T, et al. HIV infected cells are killed by rCD4-Ricin A chain. Science 1988 ; 242 : 1166-8.

23. Walker BD, Kowalski M, Chun Goh W et al. Inhibition of human immunodeficiency virus syncitium formation and virus replication by castanospermine. Proc Natl Acad Sci USA 1987 ; 84 : 8120-4

24. Richardson RC, Choppin PW. Oligopep tides that specifically inhibit membrane fusion by paramyxoviruses: Studies on the site of action. Virology 1983 ; 131 : 518-32.

25. Guntaka RV, Mahy BW, Bishop JM Varmus $H$. Ethidium bromide inhibits appearance of closed circular viral DNA and integration of virus-specific DNA in duck cells infected by avian sarcoma virus. Nature $1975 ; 253: 507-11$

26. Avery RJ, Levy JA. The effect of ethidium bromide on $C$ type virus production and induction. Virology $1979 ; 95$ : 277-84.

27. Darlix JL, Wang P, Gabus C (résultats non publiés)

28. McCune JM, Rabin LA, Feinberg MK, et al. Endoproteolytic cleavage of gpl60 is required for the activation of HIV. Cell 1988 :
Une nouvelle stratégie et deux cibles pour une chimiothérapie anti-rétrovirale

En théorie, toutes les étapes du cycle de HIV sont des cibles potentielles pour des drogues anti-rétrovirales. A ce jour, de gros efforts sont faits afin que le virus HIV ne puisse infecter les cellules, soit grâce à un leurre qui est la molécule $\mathrm{CD} 4$ recombinante (rCD4) qui se complexe aux SU gpl20 [20], soit en inhibant la réverse transcriptase par des analogues de nucléotides comme l'AZT (azidodidésoxythimidine) et le ddC (didésoxycytidine) [7].

Tant la rCD4 que les analogues de nucléotides semblent bloquer efficacement la réplication de HIV en culture de cellules (ex vivo). Cependant les résultats récents du traitement de plusieurs centaines de malades par l'AZT [9] montrent que l'efficacité de l'AZT est inégale et de courte durée. Les essais thérapeutiques viennent de commencer avec la rCD4, mais on peut craindre que celle-ci, injectée de façon répétée, induise une réponse immune chez le malade. D'ailleurs les manifestations auto-immunes ne sont pas rares chez les personnes $\mathrm{HIV}^{+}$, et elles sont probablement liées à la présence d'anticorps antiCD4 soluble (CD4s). Ces deux approches ont aussi l'inconvénient de ne pas bloquer la production de virus infectieux, et par conséquent les virus HIV matures pourront toujours provoquer la formation de syncitia par les SU gpl20, avoir des effets immunosuppresseurs par les TM gp4l, et infecter les cellules par des voies d'entrée différentes de celle impliquant la reconnaissance classique du CD4 (monocytes à macrophages, par exemple [21]). En dépit de ces limitations, ces deux approches méritent d'être poursuivies car elles correspondent à une chimiothérapie sélective. L'une inhibe les SU gpl20 et l'autre, la RTp51-p66. Mais n'oublions pas que HIV, comme les autres rétrovirus, est doué d'une dérive génétique intense, et une chimiothérapie sélective a quelques chances d'être contournée par un virus HIV mutant.

En plus de cette approche sélective, il faudrait pouvoir prendre le virus HIV de vitesse et l'annihiler dès qu'il apparaît à la surface des cellules infectées. Toute la question est de savoir comment? Faut-il radicalement tuer les cellules infectées, mais au risque d'éliminer une population cellulaire indispensable à la vie de l'organisme? ou des drogues peuvent-elles forcer les cellules $\mathrm{HIV}^{+}$à ne produire que des virions et/ou des pseudo-particules non infectieux? Cette dernière approche aurait le double mérite d'arrêter la dissémination virale et de stimuler la réponse immune chez la personne infectée.

L'approche qui consiste à tuer les cellules infectées par et produisant HIV a récemment été explorée en utilisant la toxine ricine A couplée à la rCD4 [22]. Les cellules $\mathrm{HIV}^{+}$ sont reconnues par la ricine-rCD4 grâce aux interactions rCD4-SU gpl20, puis tuées par la ricine portée par la rCD4. La question est maintenant de savoir quelles seront la tolérance et la stabilité du composé ricine-rCD4 in vivo ?

\section{La maturation des virions HIV et les} protéines NC pl5 et PR p12 comme cibles d'une nouvelle chimiothérapie anti-rétrovirale.

L'approche, moins radicale, qui est celle de laisser les cellules $\mathrm{HIV}^{+}$produire un virus non infectieux doit être explorée de deux façons complémentaires. La première consiste à empêcher l'activation des virions HIV en bloquant la maturation des précurseurs Prgag, Prgag-pol et Prenv. La maturation des Prgag et Prgag-pol est sous le contrôle de la protéase PR pl2 qui est une aspartylprotéase et qui peut être en partie inhibée in vitro par la pepstatine A [19]. Le Prenv (gpl60) est clivé sur SU gpl20 et TM gp4l sans doute par des protéases cellulaires, et ce serait probablement une cible de choix pour un agent anti-rétroviral. En effet, bloquer la maturation du Prenv par la castanospermine [23] aurait pour conséquences d'empêcher à la fois l'activation du virus et la fusion des cellules $\mathrm{HIV}^{+}$qui aboutit à leur disparition. Modifier par des oligopeptides hydrophobes l'ancrage dans la membrane cellulaire du Prenv [24], et donc sa maturation, est une autre possibilité que nous commençons à explorer.

L'approche développée au labora- 
toire et qui est complémentaire de la première, consiste à bloquer les fonctions de la protéine de nucléocapside (NC pl5). Dans le virion, la petite protéine NC est intimement associée au génome pour former avec lui une «ribonucléoparticule». Au cours de la formation de virus infectieux, cette protéine NC joue deux rôles clés, car elle gouverne l'encapsidation du génome ARN dans la capside virale, puis l'hybridation au génome de l'ARNt initiateur de la transcription inverse (figure 3) [17, 18]. Si les fonctions de NC sont bloquées, les virions produits ne devraient pas être infectieux. Alors, sachant que NC est une protéine «à doigt ${ }^{*}$, un genre sans doute très répandu dans la cellule, est-il possible d'agir et comment?

Des travaux vieux de dix ans et plus révèlent que des agents s'intercalant dans les acides nucléiques peuvent inhiber l'infection d'une cellule par un rétrovirus [8, 25]. De plus, ces agents peuvent faire chuter le titre infectieux de rétrovirus produits par des cellules chroniquement infectées. Ainsi en est-il du «bromure d'éthidium » (BET) que les cellules en culture supportent bien [26]. Un autre agent intercalant, l'actinomycine $\mathrm{D}$ qui est utilisée en chimiothérapie anticancéreuse, fait aussi chuter le titre infectieux de virus produits par des cellules infectées [8]. Nous avons examiner les effets de ces drogues sur la production, par des cellules infectées, de rétrovirus aviaire (RSV), murin (MuLV) et humain (HIV). Les résultats obtenus sont très encourageants et montrent que ces drogues inhibent la protéine NC in vitro et en culture de cellules [27]. Toutefois, l'actinomycine $\mathrm{D}$ est très cytotoxique et on ne peut guère envisager de l'utiliser comme agent anti-HIV in vivo. Le bromure d'éthidium est plus intéressant car il bloque aussi bien les fonctions de la protéine NC que celle de l'intégrase, et donc l'infectivité des virions aux stades précoce et tardif. Cependant, même si cette drogue est bien tolérée par les cellules en culture, c'est un agent mutagène.

\footnotetext{
* Voir m/s no 10, vol. 4, p. 624.
}

$\mathrm{m} / \mathrm{s} n^{\circ} 4$ vol. 5 , avril 89

\section{Conclusion}

Un des objectifs majeurs de la chimiothérapie anti-rétrovirale devrait être d'empêcher la formation de virions infectieux, qu'ils soient intraou extracytoplasmiques [11, 21]. Il faut donc bloquer la maturation des virons HIV pour que la production de HIV infectieux cesse, et ainsi arrêter tout à la fois la dissémination de HIV et les effets cytoxiques des glycoprotéines. On peut aussi espérer que les particules virales non infectieuses stimulent les défenses immunitaires contre HIV. Dans ce but, il est nécessaire de «traquer » des inhibiteurs de la protéase PR pl2 et de la NC pl5. De puissants inhibiteurs de la NC pl5 sont connus ; maintenant, nous devons en chercher d'autres, aussi efficaces mais peu ou pas cytotoxiques et mutagènes. La chasse aux inhibiteurs de la protéase PR pl2 s'annonce aussi difficile, et celle aux agents bloquant la maturation du Prenv tout autant [28]. L'avenir proche du traitement anti-HIV semble ainsi s'orienter vers l'utilisation de « cocktails » d'agents anti-rétroviraux (c'est-à-dire, AZT contre la transcriptase inverse, castanospermine contre les glycoprotéines, pepstatine-A contre la protéase et agents intercalants contre la protéine NC pl5). A plus long terme, la maturation de HIV (NC pl5 et PR pl2) sera peut-être son vrai talon d'Achille, et avec un peu de chance...

\section{Remerciements}

Le travail sur les inhibiteurs de protéines de HIV est poursuivi dans le laboratoire par Piao Wang, Caroline Gabus et Valérie Lullien. Je tiens à remercier Bernard Roques (Paris) pour ses idées très fructueuses sur les inhibiteurs de la protéine NC des rétrovirus, Pierre Tambourin (Paris), Marc Sitbon et Jean-Paul Lévy (Paris), pour la collaboration entreprise sur les effets des inhibiteurs de la NC in vivo. Le travail poursuivi dans le laboratoire bénéfice de crédits destinées à la lutte contre le SIDA (PNRS).

\section{Summary}

A new strategy and two targets for antiviral therapy in AIDS

Infection with HIV (human immunodeficiency virus) results in a severe immunosuppression due predominantly to the depletion and probably the disfunction of helper/inducer $\mathrm{T}$ lymphocytes (T4 cells). In addition monocytes and macrophages serve as a reservoir for HIV which contributes to virus dissemination by a Trojan Horse mechanism. The different stages in the life cycle of HIV present a variety of potential targets for antiretroviral agents. But one of the major goals in antiviral chimiotherapy should be to inhibit the production of infectious HIV as intra or extracellular virions. It is the purpose of this review to focus on viral assembly and on HIV virus maturation. In HIV infected cells the virion proteins are first synthesized as the polyprotein precursors Prgag, Prgag-pol and Prenv which subsequently assembled at the plasma membrane. Production of infectious virions involves both the obligatory processing of the precursors by the viral protease and by the host glycosylating enzymes and the encapsidation of replication competent HIV genomic RNA by the nucleocapsid protein. Inhibitors of the nucleocapsid protein NC pl5, the protease PR pl2 and the host glycosidases have been identified and shown to impair in vitro the production of infectious HIV virions. It is hoped that in vivo a combination of these drugs and AZT will stop both the production of infectious HIV and the cytotoxic and immunosuppressive effects of HIV glycoproteins.

\section{TIRÉS A PART}

J.-L. Darlix. 